\title{
Stool Consistency: Looking Beyond the Bristol Stool Form Scale
}

TO THE EDITOR: With great interest we have read the article of Chen et $\mathrm{al}^{1}$ on the effects of Lactobacillus casei Shirota on stool consistency in constipated patients. Apart from an improvement of constipation-related symptoms, they also showed a clear interaction between baseline Bristol stool form scale (BSFS) consistency levels and baseline relative abundance of Lachnoclostridium, Lachnospiraceae UCG-004, Pseudobutyrivibrio, and Ruminiclostridum 5. In line with this observation, an increasing number of studies use BSFS to correct for differences in fecal consistency in microbiome analyses. The generalizability of this patient-reported tool is however unclear. The BSFS has been developed in the early 90 s as a surrogate marker of whole-gut transit time (WGTT) and has been recommended for the use as such in both clinical and research settings. ${ }^{2,3}$ Although initially designed for WGTT, the BSFS is often used as a measure of stool consistency.

Recently, Blake et $\mathrm{al}^{4}$ studied the correlation between BSFS and fecal water content in 169 healthy adults and reported a high correlation $(r=0.701)$ when BSFS was assessed by trained experts. However, the correlation was only moderate $(r=0.491)$ when BSFS was self-reported, underlining the importance of interrater differences and experience when using the BSFS. The authors recommended that further research is required in order to assess reliability in different patient groups and to make comparisons with

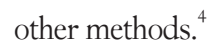

We suggest fecal dry weight content (ie, percentage dry weight after 4-5 hours vacuum drying $0.5 \mathrm{~g}$ feces at $60^{\circ} \mathrm{C}$ ) as a more objective alternative to determine stool consistency. Excellent reproducibility of this method was found by repeating the procedure for 20 samples, showing an intraclass correlation coefficient of 0.948 . We then compared patient-reported BSFS with fecal dry weight content in a pooled dataset including 122 stool samples of healthy volunteers and 67 samples of irritable bowel syndrome patients. We found a correlation of -0.411 in the total dataset, with a lower correlation in healthy subjects $(r=-0.337)$ compared to irritable bowel syndrome $(r=-0.536)$. Furthermore, in a subgroup of healthy elderly $(70-85$ years, $n=21)$ the correlation was -0.233 , pointing towards possible difficulties in rating stool consistency within specific subgroups.

In conclusion, we consider the BSFS to be a helpful tool in daily clinical practice as it is a quick and easy patient-reported measure of WGTT, and can be used as a surrogate marker for stool consistency. However, one should be aware of the relatively low correlation between the BSFS and more objective measures of stool consistency, and the considerable inter-rater error when using the BSFS. Therefore, when analyzing stool consistency in scientific studies, we suggest considering more objective assessments such as fecal dry weight or water content.

Lisa Vork, ${ }^{1}$ Ellen Wilms, ${ }^{1 *}$ John Penders, ${ }^{2}$ and Daisy M A E Jonkers ${ }^{1}$ 'Division Gastroenterology-Hepatology, Department of Internal Medicine, ${ }^{2}$ Department of Medical Microbiology, NUTRIM School of Nutrition and Translational Research in Metabolism, Maastricht University Medical Center+, Maastricht, the Netherlands

1. Chen S, Ou Y, Zhao L, et al. Differential effects of Lactobacillus casei strain Shirota on patients with constipation regarding stool consistency in China. J Neurogastroenterol and Motil 2019;25:148-158.

2. Lewis SJ, Heaton KW. Stool form scale as a useful guide to intestinal transit time. Scand J Gastroenterol 1997;32:920-924.

3. Heaton KW, O'Donnell LJ. An office guide to whole-gut transit time. Patients' recollection of their stool form. J Clin Gastroenterol 1994;19:2830.

4. Blake MR, Raker JM, Whelan K. Validity and reliability of the Bristol Stool Form Scale in healthy adults and patients with diarrhoea-predominant irritable bowel syndrome. Aliment Pharm Ther 2016;44:693-703.

Financial support: None.

Conflicts of interest: None.

Author contributions: Lisa Vork and Ellen Wilms: perform experiments, data collection, data analysis, and drafting and finalising manuscript; and John Penders and Daisy M A E Jonkers: critical revision of the manuscript for important intellectual content, and finalizing the manuscript. 